\title{
OVERVIEW OF MAIN-MECHANICAL- COMPONENTS AND CRITICAL MANUFACTURING ASPECTS OF THE WENDELSTEIN 7-X CRYOSTAT
}

\author{
Torsten Koppe, Dr. A. Cardella, , B. Missal, B. Hein, \\ R. Krause, H. Jenzsch \\ Max-Planck-Institut für Plasmaphysik, \\ EURATOM Association \\ D-17491 Greifswald, Germany \\ torsten.koppe@ipp.mpg.de
}

\author{
Jens Reich \\ ITER Organization \\ 13067 St Paul lez Durance Cedex, France
}

Wendelstein 7-X (W7-X) will demonstrate the possibility of a stellarator for a future fusion power plant. This stellarator fusion experiment is at present in the assembly phase at the MaxPlanck-Institut für Plasmaphysik (IPP). The main advance of the static plasma is caused by the three dimensional shape of the coils. But inside the Cryostat this extravagant geometry of the coils efforts also a three dimensional contour of the main mechanical components. One of the ambitious challenges is how to build up such complex machine. The manufacturing of these complex devices have been demanded the newest manufacturing methods. At 2014 Wendelstein 7-X will be the world's largest superconducting helical advanced stellarator. The toroidal plasma vessel geometry follows exactly the three dimensional shape of the plasma. It contains the plasma with a great diameter of $11 \mathrm{~m}$ and an average plasma diameter of $1.1 \mathrm{~m}$. To control the plasma geometry it is necessary that all the 20 planar and 50 non planar coils are not only extreme narrow positioned to the Plasma Vessel but also within a tolerance of $1.5 \mathrm{~mm}$ to each other. To meet this requirement and to withstand the high magnetic forces a complex coil support structure was created. The Central Support Ring have to bear the coils but the different inter coil supports canalize the forces by very stiff connections on one side and sliding areas on the other side. The coils and the support structure are enclosed within the Outer Vessel with its domes and openings. The Outer Vessel, the Plasma Vessel and the ports generate the boundaries for the Cryostat. The vacuum inside provides thermal insulation of the magnet system which is cooled down to $4 \mathrm{~K}$. The 254 ports secure the access to the Plasma Vessel with all the supply lines and the diagnostics. Due to the different thermal movements the Plasma Vessel, Outer Vessel and the Central Support Ring have to be supported separately. The Central Support Ring is held by 10 cryo legs. The Plasma Vessel supporting system is divided into two separate systems, allowing horizontal and vertical adjustments to centre the Plasma Vessel during thermal expansion. Beside an overview about the main components in the cryostat like the plasma vessel, the outer vessel, the ports and the different support systems this paper describes the most demanding manufacturing methods. The author delineates some disparate and special problems during the manufacturing of the components at the companies in the different European countries.

Keywords Wendelstein 7-X; Cryostat; Plasma Vessel; Outer Vessel; Coil Support Structure, Ports

\section{INTRODUCTION}

Wendelstein 7-X is presently under construction at the Max-Planck-Institut for Plasmaphysics in Greifswald, Germany. With the completion in 2014 a big scientific and technological step will be reached, because W7-X is designed for high-power steady-state operation up to $30 \mathrm{~min}$ plasma operation at $10 \mathrm{MW}$ ECRH. But also Wendelstein $7-\mathrm{X}$ is a first-of-a-kind development. A lot of new technologies were developed and many uncertainties and surprises were endured.

Wendelstein $7-\mathrm{X}$ with the $30 \mathrm{~m}^{3}$ plasma volume is a big machine. The internal vacuum vessel called 'Plasma Vessel' (PV) provided the first barrier for the ultra-high vacuum $\left(\leq 10^{-}\right.$ ${ }^{8}$ mbar). To control the plasma form it is necessary that all the 20 planar and 50 non planar coils are positioned within a tolerance of $1.5 \mathrm{~mm}$. To meet this requirement a complex coil support structure was created. The superconducting magnet system, the coil support structure, the liquid helium cooling pipes and the thermal shield are enclosed between the Plasma Vessel and a second external toroidal vacuum vessel, the 'Outer Vessel' (OV). An intermediate vacuum $\left(\leq 10^{-6} \mathrm{mbar}\right)$ is established between the vessels. In this kind $\mathrm{W} 7-\mathrm{X}$ is a big cryogenic device with a cold mass of $425 \mathrm{t}$. The boundaries of the cryostat are formed by the Plasma Vessel, the Outer Vessel and the different ports. The ports will join the Plasma Vessel through the cryostat coming from the Outer Vessel. Also the different support structures for the Plasma Vessel and the Coil Support Structure have to lead through the cryostat.

For technological aspects the entire W7-X machine is divided in five similar modules. Each module consists of two 
flip symmetrical half modules. So it was possible to manufacture up to ten equal parts and connect them to bigger components.

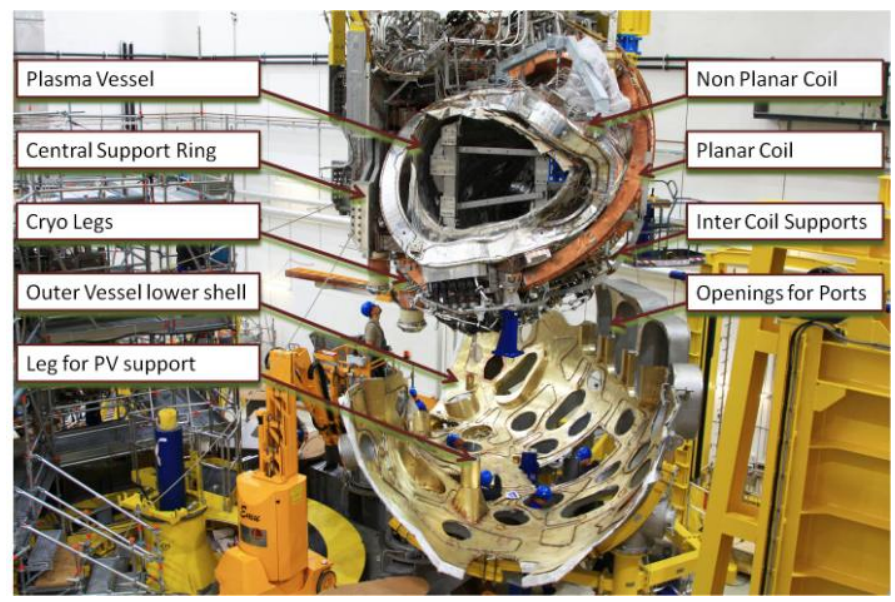

Figure 1. Main components shown at first module of the Wendelstein 7-X

\section{OUTER VESSEL}

Also the Outer Vessel (OV) consists of 5 similar modules, but as an exception the OV is divided in an upper and lower shell. The Vessel is designed as a torus with an outer diameter of approximately $16 \mathrm{~m}$. The internal diameter of the cross section is $4.4 \mathrm{~m}$. It is made of austenitic steel 1.4429 (X2CrNiMoN1713-3) and the wall thickness is $25 \mathrm{~mm}$. At the inner surface of the Outer Vessel an active cooled thermal radiation shield with the super isolation is implemented. In addition the thermal insulation of the magnet system is provided by the high vacuum. The access to the Plasma Vessel or the cryostat is achieved via ports and domes. 524 domes were welded onto the torus [1]. The Outer Vessel is borne by 15 supports, which are directly connected to the machine base.

Five toroidal sectors were welded together to form one module shell. The 524 openings were cut and completed with their domes precisely (Figure 2) with the aid of three dimensional measurements. But one of the greatest difficulties during the manufacturing and assembly of the Outer Vessel were the weak structure of the separate shells despite of the 25 $\mathrm{mm}$ wall thickness. Therefore different stiff workarounds had to be created to hold on the achieved geometry at the separate assembly steps.

Now all modules of the Outer Vessel are not only manufactured. At present all modules have been insulated with the thermal isolation and four were assembled on the machine base. The welding of the first module section between module 5 and 1 is planned for autumn 2011. The manufacturer is MAN DT GmbH.

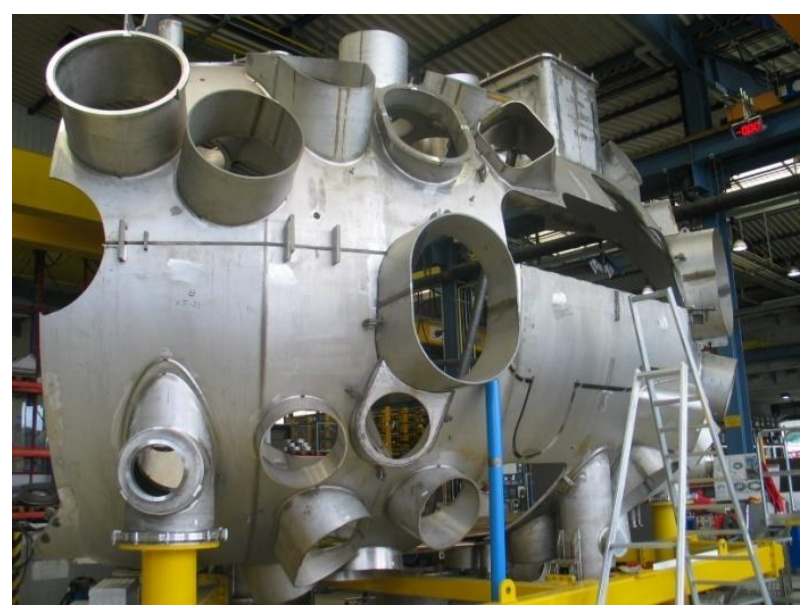

Figure 2. Mounting of the domes

\section{PORTS}

The easy function of the 254 ports is to connect the plasma vessel through the cryostat with the environment. But the thermal movement between the Plasma Vessel and the Outer Vessel requires that all ports are equipped with bellows which can vary between $100 \mathrm{~mm}$ circular to 1170 x $570 \mathrm{~mm}$ rectangular sizes. The type one ports consists of two pipes which are connected with the bellows in between. Most of them will be used for the several diagnostics which are planned to install. Therefore this group is also called diagnostic ports. Also the great rectangular and special ports are type one ports. They will contain the heating systems like ECRH, ICRH or NBI and will allow maintenance. The type two ports are made of only one pipe. One plate at the port and one at the Outer vessel are connected with the bellow so that thermal movement is also tolerable. This so called supply ports will be used for the supply lines of the in-vessel components.

During the manufacturing of the ports at the Swiss company Romabau-Gerinox AG some critical points had to be passed. Not only the manufacturing of the different types of bellows at the Swiss company Kompaflex AG or their different stiffness and spring rate was sensible, but also the protecting during the further manufacturing processes and the assembly at W7-X had to be respected. Furthermore the cleanness after the manufacturing and during the long time of storing and preparing for the assembly was a topic. At least the tolerance chain and the achievable accuracy during the manufacturing and the assembly inside the cryostat were underestimated.

At present the port preparation for the assembly is running well. Each port will be covered by an own thermal insulation. The assembly at three modules is on track (see Figure 3). 


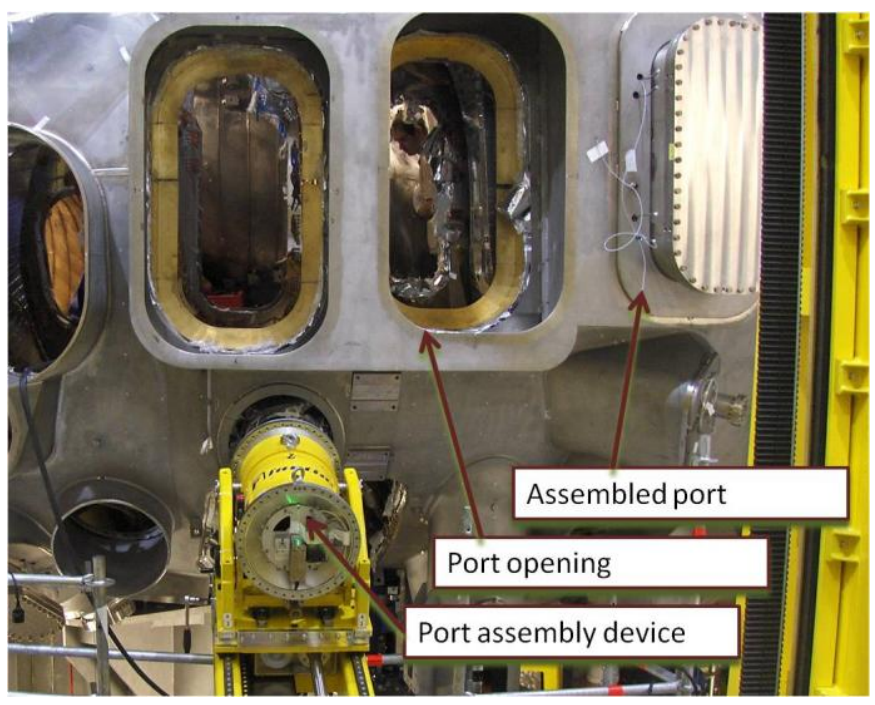

Figure 3. Port assembly

\section{PLASMA VESSEL AND THEIR SUPPORTS}

\section{A. Plasma Vessel}

Five similar Plasma Vessel (PV) modules are joined together along radial-poloidal and radial-toroidal planes. The shape of the Plasma Vessel cross-section changes within each module from a triangular to a bean form and back again to a triangular form. Because of this complicated form, the surfaces switch between convex and concave. The maximum outer diameter of the torus is approximately $12 \mathrm{~m}$; the minimum inner diameter is $8 \mathrm{~m}$. The wall is made of the austenitic steel 1.4429 (X2CrNiMoN17-13-3) and has a thickness of $17 \mathrm{~mm}$ [2]. The inner surface will be protected against the plasma heat by in-vessel components. On the outside are welded cooling pipes (see Figure 4). The vessel and pipes are covered with the thermal shield consisting of an actively cooled glass fibred shield and copper shell with multilayer super insulation which minimizes the thermal radiation from the Plasma Vessel to the coils.

The supplier MAN DT GmbH (Deggendorf, Germany) manufactured and delivered the Plasma Vessel parts and connected components. The assembly of the thermal insulation and the welding to modules is finished. The next critical point is the welding of the first two modules together in autumn this year.

\section{B. Plasma Vessel support system}

During various load-cases the axial and lateral stiffness of all bellows of the ports will create a resulting spring-force which acts directly on the plasma vessel supports.

The plasma vessel supporting system is divided into two separate systems allowing horizontal and vertical adjustments to centre the plasma vessel during the thermal expansion. The vertical supports consist of three pendulum supports per module. They have to withstand and transfer the forces to the machine base. Simultaneously the horizontal support system is centring the Plasma Vessel. To close the gaps to the cryostat there are used bellows.

Also all Vertical Plasma Vessel Supports were delivered by MAN DWE GmbH and for all modules they are implemented. The parts for the horizontal system are manufactured but not yet assembled.

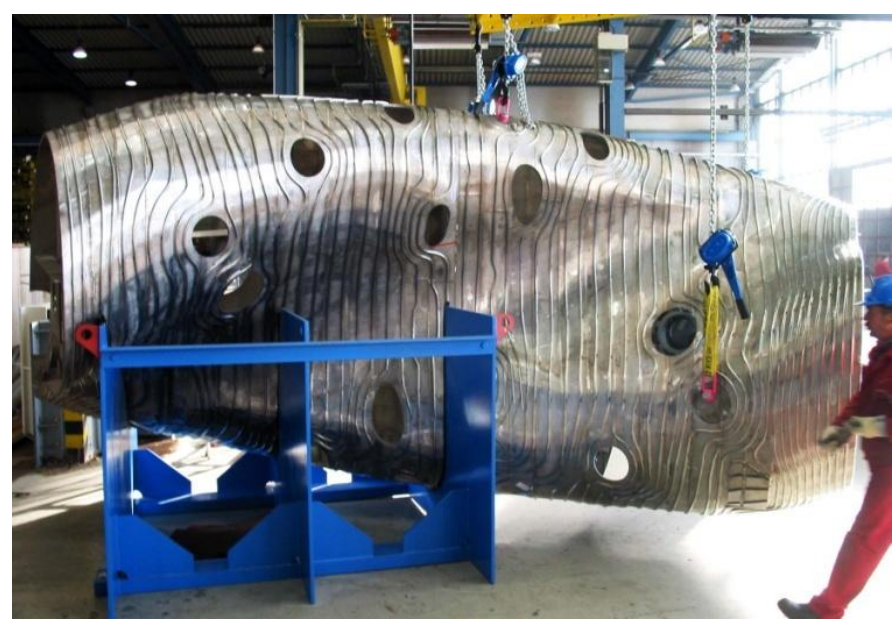

Figure 4. Plasma Vessel section

\section{SUPPORT STRUCTURE OF THE MAGNETIC SYSTEM}

\section{A. Overview}

While the assembly special elements were used to reach the tight tolerances of $1.5 \mathrm{~mm}$. The entire Coil Support Structure (CSS) saves this configuration of the coils during operation. The Coil Support Structure consists of several types of structural components. In the middle is composed the strongest part the Central Support Ring. All the superconducting coils are connected by the Central Support Elements (CSE) to the Central Support Ring. The ring itself is supported by 10 cryo legs which allow a thermal movement. Between the coils are the different types of supports situated. The CSS is subjected to various loads during the life of the $\mathrm{W} 7-\mathrm{X}$ experiment. The main loads are generating during coil de-energisation and subsequent magnetic forces. Additional loads can occur due to cooling down and the dead weight of the CSS. All of these loads were taken into account in the structural analysis of the CSS. The Cryostat encloses this complex structure. Together with the active cooled thermal shield at the inner surfaces of the cryostat boundaries it is saved that the entire structure can reach the $4 \mathrm{~K}$ temperature.

\section{B. Central support ring}

The Central support ring is divided into five modules. Each module is split in two equal half modules. The ring is made of a mix of 1.3960 casting and 1.4429 steel plates welded together. The ring has two main flange interfaces that connect the individual CSR half-modules. Both ring parts of one module are connected by a step flange. The half module flange is bolted by standard bolts and some shear bolts to avoid movements during operation. At the module section a flat flange configuration is used to connect the modules. Due to 
the risk of a flange face misalignment after the positioning of neighboring models, shear bolts cannot be used with the module flange. Instead shim plates and diamond foils will conserve the achieved position [3].

During the first period of manufacturing additional loads were discovered by enhanced Finite Elements Models. This circumstance leads to exceptional numerous of changes concerning the central support ring. Therefore the time of manufacturing elongated uncommonly, this evolves into some critical situations. The terms of contract and the technical specification hadn't foreseen a so long time of contract. Most of the drawings had to adapt several times. And the tighter manufacturing tolerances lead to a special mechanical machining. In addition the needed liquids during the milling polluted hidden surfaces. The cleaning demanded a exceptionally effort.

But at the end all modules were completed and delivered to IPP. They are successful assembled with the coils meeting the also tight assembly tolerances. The ten half modules of the CSR are built by the Spanish company Equipos Nucleares SA, Maliano. The mechanical machining was carried out by the Italian company CLP (Borgo san Dalmazzo, Italy).

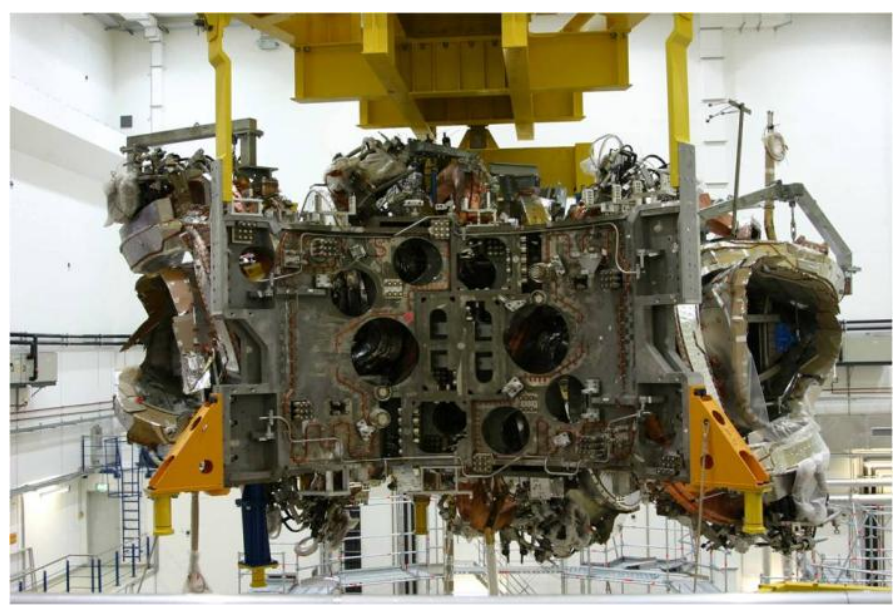

Figure 5. Central support ring

\section{Inter coil supports}

In order to resist the high magnetic forces during operation the coils support each other by a number of different support elements. 60 Lateral Support Elements (LSE) connect the coils on the outside of the magnetic system. LSE interconnect the non planar coils on the outboard side and can transfer both forces and moments in all directions. The LSE add toroidal stiffness to the torus and thus limit deflections at the far end of the non planar coil. The 245 Narrow Support Elements (NSE) will endure the high magnetic forces on the inner side and at the smallest gaps [4]. The 80 Planar Support Elements (PSE) are the link between the planar and non planar coils. Due to the higher flexibility of the planar coils the PSE were introduced to limit the deflection during operation. There are two main types of PSE a rigid bolted connection and a contact support. The 20 Contact Elements (CE) are placed between the coils at the half module and module junction [4].

All these Inter Coil Supports are small but complex mechanical structures. It was needed a not normal E-beam welding at the LSE and PSE which required a special welding procedure qualifications. Furthermore the mechanical machining of the LSE had to adapted to the three dimensional measurement during the assembly. And the NSE got a special coating at the contact surfaces. All these extraordinary efforts led to a high logistical and technical support.

All Inter Coil Supports were delivered and assembled except the Inter coil supports at the module junction.

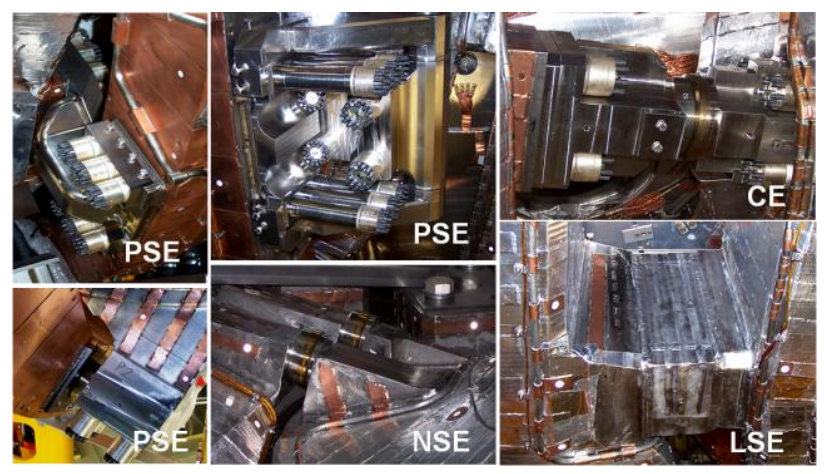

Figure 6. Different inter coil supports of Wendelstein 7-X

\section{Cryo Legs}

The Cryo legs support the central ring with the connected coils on the machine base. Each module is held by two Cryo Legs. The Cryo Legs are partially located within the Cryostat and partially outside. Therefore a proper insulation to prevent heat transfer into the Cryostat is needed. This is achieved by introducing a glass fibred reinforced plastic (GFRP) pipe between the steel sections of the Cryo Leg. The Cryo Leg has to be able to balance the movements caused by the cooling down of the magnetic system. This shrinkage is allowed by the use of sliding bearings between Cryo Leg and machine base. In addition a callot bearing is used to eliminate transfer of moments to the Cryo Leg base. At the section to the environment a bellow provides the cryostat vacuum. The Cryo Leg is loaded with the deadweight of the CSS. In addition the Cryo Leg sees loads during operation as a result of CSR deformation, which again is caused by the de-energized coils. The glass fibred reinforced plastic pipe is fitted into a stainless steel ring on the outer ends of the tube [5].

Partly the difficulties were caused by the design and also logistical problems occurred. To avoid defects due to different thermal movements of the stainless steel flange and the fibred reinforced plastic pipe a special analyzes was developed. The results were implemented in the design of the cryo legs and other components. At the end five companies from four European countries were involved in the developing and manufacturing of the entire cry leg. 
All Cryo Legs were delivered by Ettore Zanon (Schio, Italy). The glass fibre reinforced plastic pipes were produced and thoroughly checked by IMA GmbH (Dresden, Germany). All modules are fit out with the cryo leg and their bellows.

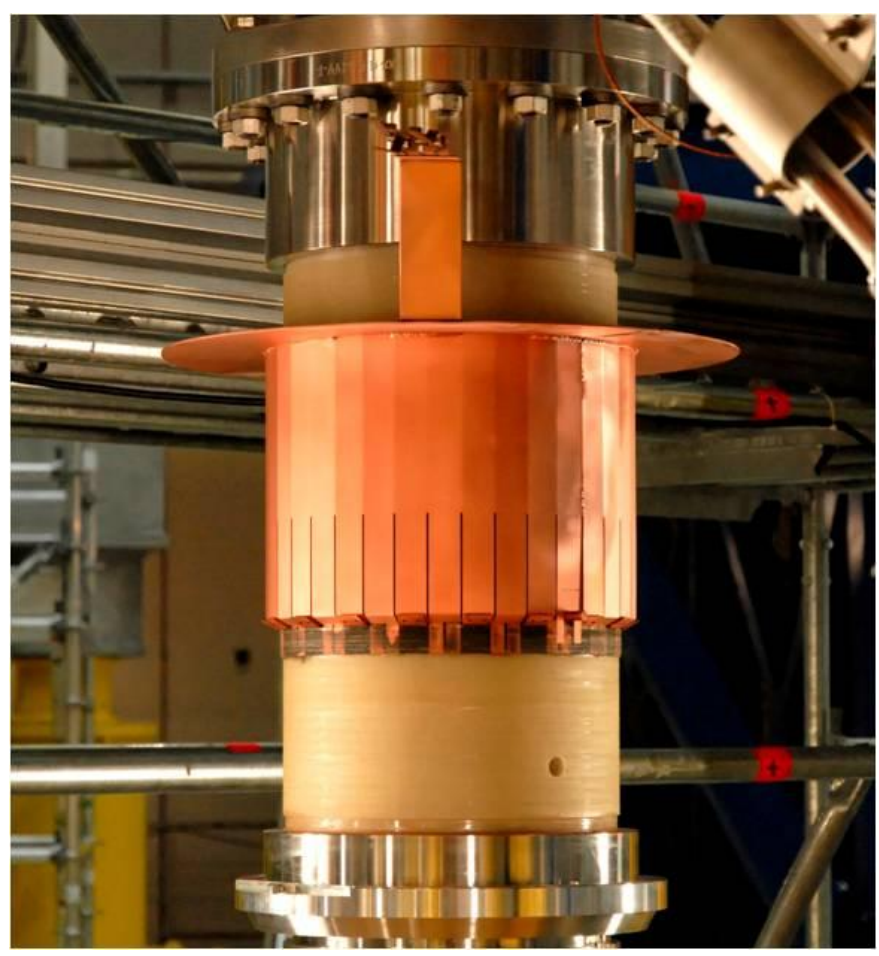

Figure 7. Cryo leg without the bellow and the bearings

\section{CONCLUSION}

The manufacturing of the main Cryostat components is coming to an end. The new experiences during the manufacturing process were manifold and sometimes unexpected. In general it can be assumed that the most important arrangement has to define adequate precisely in a very early period of the providing of a known component. A technical specification and a contract with a supplier is the result after this period. The difficulty in this is to find the right balance between the details and the overview. A further experience has to do with the monitoring. It could be shown that a tight monitoring at the manufacturer is necessary to get good results. And last but not least it was discovered, that sometimes ostensible small components can require an unusual effort. In opposite some big components were easy to handle.
Nevertheless the assembly is well in progress. It could be shown that almost the assembly requirements were achieved. In general it can be pointed out that all parts which are named in this paper are on the recent internal time schedule. From this point of view there are no doubts to complete the assembly up to 2014. But because of the complexity of the parts and the tight tolerances the production process demanded a great challenge to the contractors.

\section{ACKNOWLEDGMENT (HEADING 5)}

????

\section{REFERENCES}

The template will number citations consecutively within brackets [1]. The sentence punctuation follows the bracket [2]. Refer simply to the reference number, as in [3] - do not use "Ref. [3]" or "reference [3]" except at the beginning of a sentence: "Reference [3] was the first ..."

Number footnotes separately in superscripts. Place the actual footnote at the bottom of the column in which it was cited. Do not put footnotes in the reference list. Use letters for table footnotes.

Unless there are six authors or more give all authors' names; do not use "et al.". Papers that have not been published, even if they have been submitted for publication, should be cited as "unpublished" [4]. Papers that have been accepted for publication should be cited as "in press" [5]. Capitalize only the first word in a paper title, except for proper nouns and element symbols.

For papers published in translation journals, please give the English citation first, followed by the original foreign-language citation [6].

[1] F. Schauer, Status of Wendelstein 7-X Construction, proc. 24th SOFT, Warshaw 11-15/9/2006

[2] A. Cardella, Construction of the Vacuum Vessels and the Magnet Supporting, Structures of WENDELSTEIN 7-X, proc. 24th SOFT, Warshaw 11-15/9/2006

[3] [3] J. Reich, Manufacture of Cryostat components for Wendelstein 7 X, Fusion Eng. Des. 69 (2003) 345-348

[4] [4] R. Krause, Spezifikation NSE Halbzeuge, Technical Specification, 1-AAA00HH5 S-0000.1, IPP documentation, 28.07.2006

[5] [5] H. Jenzsch, Test von LSE Dummies, Specification, 1-AAA00HH0 S-0000.0, IPP documentation, 10.02.2006 